\title{
Aspectos clínicos e inmunológicos de la infección por SARS-CoV-2
}

\section{Clinical and immunological aspects of SARS-CoV-2 infection}

\author{
Nataniel-A Chaparro-M ${ }^{1}$; Alex-O Franco-L ${ }^{2}$
}

Forma de citar: Chaparro NA, Franco AO. Aspectos clínicos e inmunológicos de la infección por SARS-CoV-2. Salud UIS. 2020; 52(3): 295-309. doi: http://dx.doi.org/10.18273/revsal.v52n3-2020010 (c) (i)

\section{Resumen}

Objetivo: describir los aspectos moleculares y celulares de la respuesta inmune frente a SARS-CoV-2; y las repercusiones clínicas, producto de mecanismos inmunes. Introducción: la aparición de una neumonía atípica en China, en diciembre de 2019, provocó un confinamiento global. El agente responsable de esta enfermedad fue nombrado por el Comité Internacional de Taxonomía de Virus como SARS-CoV-2, y la enfermedad que produce fue denominada COVID-19 por la OMS el 11 de febrero del 2020. Metodología: para este estudio descriptivo se indagó en la base de datos de Pubmed, Science, Nature, The Lancet, The New England Journal of Medicine, medRxiv y Google académico; cuyos descriptores utilizados fueron COVID-19, 2019-nCoV, SARS-CoV-2, linfocitos, anticuerpos e inmunidad; incluyendo en la revisión 130 estudios. Resultados: las manifestaciones clínicas más frecuentes que produce SARS-CoV-2 son: fiebre, tos seca y fatiga; siendo los individuos ancianos que presentan con mayor frecuencia complicaciones como el síndrome de distrés respiratorio agudo, arritmias, fallo cardíaco agudo y shock séptico. Se identificó descensos significativos de las células NK, linfocitos B, linfocitos T CD4+ y CD8+ en sangre periférica en pacientes con cuadros moderados y severos de COVID-19. Discusión: Las células NK y macrófagos se encargan de la contención y eliminación viral en las primeras etapas de la infección por SARSCoV-2. No está claro la relevancia de la respuesta humoral en la contención, y eliminación de SARS-CoV-2. La respuesta de linfocitos $\mathrm{T}$ citotóxicos es esencial para la completa resolución de la infección por SARS-CoV-2, ya que logran la eliminación de las células infectadas. No existe un tratamiento antiviral específico recomendado para COVID-19, y actualmente no existe vacuna disponible.

Palabras clave: Inmunidad; Linfocitos; SARS-CoV-2; Coronavirus; COVID-19; Infección; Manifestaciones clínicas; Terapia.

\begin{abstract}
Objective: To describe the molecular and cellular aspects of the immune response against SARS-CoV-2 and the clinical repercussions resulting from inefficient immune mechanisms. Introduction: The emergence of an atypical pneumonia in China in December 2019 led to a global confinement. The agent responsible for this new disease was named SARS-CoV-2 by the International Committee on Taxonomy of viruses, and the disease it produces was named COVID-19 by the WHO on February 11, 2020. Methodology: For this descriptive study we researched the databases of Pubmed, Science, Nature, The Lancet, The New England Journal of Medicine, medRxiv and

1. Hospital Clínico Viedma. Cochabamba, Bolivia.

2. Hospital Universitario San Juan de Dios. Santa Cruz de la Sierra, Bolivia.

Correspondencia: Nataniel Aldo Chaparro Mérida. Dirección: Calle Venezuela y German Urquidi, Cbba-Quillacollo, Bolivia. Teléfono: +59177439845 Correo: nathaniel.iav.firts@hotmail.com
\end{abstract}


Google Scholar; which descriptors used were: COVID-19, 2019-nCoV, SARS-CoV-2, lymphocytes, antibodies and immunity; including 130 studies in the review. Results: The most common clinical manifestations produced by SARS-CoV-2 are: fever, dry cough and fatigue, being the elderly people who are mostly having complications such as acute respiratory distress syndrome, arrhythmias, acute heart failure and septic shock. Significant decreases in NK cells, B lymphocytes, CD4+ and CD8+ T lymphocytes were identified in peripheral blood in patients with moderate and severe COVID-19 conditions. Discussion: NK cells and macrophages are responsible for viral containment and elimination in the early stages of SARS-CoV-2 infection. The relevance of the humoral response in the containment and elimination of SARS-CoV-2 is unclear. The cytotoxic T lymphocyte response is essential for the complete resolution of SARS-CoV-2 infection, as they achieve the elimination of infected cells. There is no specific antiviral treatment recommended yet for COVID-19, and there is currently no vaccine available.

Keywords: Immunity; Lymphocytes; SARS-CoV-2; Coronavirus; COVID-19; Infections; Signs and symptoms; Therapy.

\section{Introducción}

En diciembre de 2019 se evidenció la aparición de una serie de casos de neumonía en la ciudad de Wuhan, capital de la provincia de Hubei, China; algunos de los cuales fueron inicialmente relacionados al mercado mayorista Huanan de mariscos, pescados y animales vivos, este es el lugar probable de origen del agente causal de esta enfermedad ${ }^{1}$. Este nuevo microorganismo perteneciente a la subfamilia de los Beta-coronavirus, fue completamente secuenciado por científicos chinos el 7 de enero, y nombrado como nuevo coronavirus 2019 (2019-nCoV, por sus siglas en inglés) por la Organización Mundial de la Salud (OMS) el 12 de enero de 2020; posteriormente el grupo de estudio de coronavirus del Comité Internacional de Taxonomía de Virus (ICTV, por sus siglas en inglés) asignó a este agente como Coronavirus 2 del síndrome respiratorio agudo severo (SARS-CoV-2, por sus siglas en inglés) y la enfermedad que produce fue denominado por la OMS el 11 de febrero como COVID-19 (es el acrónimo en inglés, enfermedad por coronavirus de 2019) ${ }^{2,3}$. La rápida propagación de SARS-CoV-2 en los diversos continentes; con un elevado número de personas infectadas y fallecidas, provocó que la OMS declare a la COVID-19 como pandemia global el 11 de marzo ${ }^{4}$.

\section{Características de los coronavirus}

Los coronavirus $(\mathrm{CoV})$ son virus cuyo genoma consiste en una única molécula de ácido ribonucleico (ARN) en sentido positivo, no segmentados, envueltos, son redondos y a veces pleomórficos, a los coronavirus se les dio su nombre en función de su característica en forma de corona bajo un microscopio electrónico, diámetros de 80-120 nm y con un genoma que varía de 26 a 32 kilobases, es el genoma de ARN viral más grande ${ }^{5,6}$.
El virión tiene una nucleocápside compuesta de ARN genómico y la proteína nucleocápside $(\mathrm{N})$ fosforilada, que está cubierta dentro de las bicapas fosfolipídicas y está cubierta por dos tipos de espiga: el recortador de glucoproteína de espiga (S) que se encuentra en todos los CoV, y la hemaglutinina-estereasa (HE) que existe en algunos CoV. La proteína de membrana (glicoproteína transmembrana de tipo III o M) es la proteína más abundante de la partícula viral, y la proteína de la envoltura (E) que se encuentra entre las proteínas $\mathrm{S}$ en la envoltura del virus ${ }^{7}$.

Los coronavirus son miembros de la subfamilia Orthocoronavirinae de la familia Coronaviridae, y del orden Nidovirales (ICTV). La subfamilia de los CoV se divide de forma genotípica y serológica en cuatro géneros los CoV alfa $(\alpha)$, beta $(\beta)$, gamma $(\gamma)$ y delta $(\delta)$. El $\beta-\mathrm{CoV}$ se puede clasificar en cuatro linajes virales, a saber, el linaje $\mathrm{A}-\mathrm{D}^{7,8}$. Las infecciones humanas son causadas por $\alpha$ y $\beta-\mathrm{CoV}$, pero también estos virus infectan a mamíferos y aves. Los $\beta$-CoV son endémicos en todo el mundo y representan del $10 \%$ al $30 \%$ de infecciones del tracto respiratorio superior en adultos?.

Existen siete 7 tipos de coronavirus que han sido identificados por el Centro y control de prevención de enfermedades (CDC, por sus siglas en inglés), que incluyen 2 grupos. Los coronavirus humanos (HCoV, por sus siglas en inglés) comunes: 229E, NL63 ( $\alpha$-coronavirus), OC43, HKU1 ( $\beta$-coronavirus) y otros coronavirus humanos: Coronavirus causante del Síndrome Respiratorio del Medio Oriente (MERS$\mathrm{CoV}$, por sus siglas en inglés), Coronavirus causante del Síndrome respiratorio Agudo Severo (SARS-CoV, por sus siglas en inglés) y Coronavirus 2 causante del Síndrome respiratorio Agudo Severo (SARS-CoV-2, por sus siglas en inglés), anteriormente denominado 2019-nCoV; estos tres últimos pertenecen al grupo de $\beta-\mathrm{CoV}^{9,10}$. 
Los cuatro CoV (229E, NL63, OC43 y HKU1) son prevalentes y típicamente causan síntomas de resfriado común en individuos inmunocompetentes, y algunas veces pueden ocasionar infecciones graves en poblaciones de edades extremas ${ }^{11}$. Las otras cepas, el SARS-CoV y MERS-CoV, son de origen zoonótico y se relacionan con enfermedades a veces fatales. El SARS$\mathrm{CoV}$ fue el agente causal de los brotes de síndrome respiratorio agudo severo en 2002 y 2003 en la provincia de Guangdong, China; MERS-CoV fue el patógeno responsable de brotes de enfermedades respiratorias graves en 2012 en el Medio Oriente ${ }^{12}$. Dada la alta prevalencia y amplia distribución de los coronavirus, la gran diversidad genética y la recombinación frecuente de sus genomas, y el aumento de las actividades de interfaz entre humanos y animales, es probable que emerjan periódicamente nuevos coronavirus en humanos, debido a infecciones frecuentes entre especies y eventos de contagio ocasionales ${ }^{8,10,13}$.

Tras la secuenciación del genoma del SARS-CoV-2 y $\beta$-CoV/RaTG/2013, ambos virus están en el mismo grupo y mostró un $96,2 \%$ de identidad de secuencia del genoma global, lo que sugiere que el $\mathrm{CoV}$ de murciélago y el SARS-CoV-2 podrían compartir el mismo ancestro común ${ }^{14}$. Estudios recientes destacaron que los genes SARS-CoV-2 comparten $<80 \%$ de identidad de nucleótidos y $89,10 \%$ de similitud de nucleótidos con genes SARS-CoV ${ }^{15,16}$.

\section{Epidemiología}

La infección aguda atípica del tracto respiratorio estalló por primera vez en la ciudad Wuhan, provincia de Hubei de China, desde el 12 de diciembre de 2019. El 31 de diciembre de 2019, la comisión Municipal de Salud y Sanidad de Wuhan informó sobre un grupo de 27 casos de neumonía de etiología desconocida, con una exposición común a un mercado mayorista de marisco, pescado y animales vivos, como aves de corral, murciélagos, marmotas y serpientes (denominado mercado húmedo). El 7 de enero de 2020, las autoridades chinas identificaron como agente causante del brote a un nuevo coronavirus que posteriormente fue denominado SARS-CoV-2 ${ }^{17,18}$.

Los CoV ocasionan infecciones en los seres humanos y en una variedad de animales, incluyendo aves y mamíferos como camellos, gatos, cerdos y murciélagos. Se trata de una enfermedad zoonótica, lo que significa que pueden transmitirse de los animales a los humanos ${ }^{19,20}$.
Varios estudios sugieren que el murciélago puede ser el reservorio potencial de SARS-CoV-2, y el pangolín el hospedero intermediario, sugiriendo que este es el mecanismo de transmisión al hombre, lo que produjo que COVID-19 se extienda en toda China y varios países a nivel mundial, por lo cual la OMS declaró este brote una emergencia de salud pública de interés internacional el 30 de enero de $2020^{21,22}$.

En 2003 SARS-CoV presentó una tasa de letalidad del $10 \%$ y desde entonces no se volvió a detectar en humanos. Por otra parte, se notificó que en 2013 MERSCoV alcanzó el 34\% de letalidad, y hasta el 11 de mayo de 2020 en Europa la tasa de letalidad de SARS-CoV-2 fue de $4,5 \%{ }^{23,24}$.

La transmisión de persona a persona de SARS-CoV-2 fue evidenciada por los primeros estudios efectuados en China, donde establecen una media del periodo de incubación de 5 días, con un rango de 1-14 días ${ }^{25-27}$. La transmisión por microgotas de la vía aérea, y el contacto con fómites contaminados fue dilucidada por las investigaciones de Holbrook, et $\mathrm{al}^{28}$. Existe controversia sobre si SARS-CoV-2 pueda transmitirse de una madre infectada a su producto antes del nacimiento ${ }^{29}$. Aunque la transmisión vertical no ha sido demostrada en algunos estudios efectuados en distintos países (Irán, Estados Unidos y China) ${ }^{30-32}$. Recientemente el estudio de Long, et al. determinaron que la mediana de duración de la eliminación de SARS-CoV-2 (intervalo entre el primer y el último hisopado nasofaríngeo positivo) en individuos asintomáticos fue de 19 días; aunque no definieron la carga viral respiratoria asociada con infección ${ }^{33}$.

\section{Características clínicas y complicaciones ocasionadas por SARS-CoV-2}

Los coronavirus que tienen capacidad infecciosa en el humano son siete, de estos los HCov 229E, NL63, OC43, y HKU1 son endémicos globalmente causando infecciones del tracto respiratorio superior no severas; siendo las infecciones zoonóticas producidas por SARS$\mathrm{CoV}$, MERS-CoV y SARS-CoV-2 las que producen cuadros severos de neumonía que conducen a distrés respiratorio agudo, shock séptico, fallo multiorgánico y la muerte en los individuos afectados ${ }^{34,35}$.

El nuevo patógeno SARS-CoV-2 tiene una elevada tasa de transmisión de persona a persona, los valores reproductivos básicos (R0) de COVID-19, es de 2 a 2,5, lo que indica que un paciente puede transmitir a otras 
dos o tres personas ${ }^{36}$; siendo la mayoría de los individuos infectados asintomáticos; ciertamente, en medio de la pandemia de COVID-19, los grupos vulnerables son personas sin hogar o desamparadas, sino también personas de un gradiente de grupos socioeconómicos que podrían tener dificultades para hacer frente de forma financiera, mental o físicamente con la crisis; presentando cuadros más severos los ancianos, personas con comorbilidades asociadas: hipertensión arterial, enfermedades cardiovasculares, diabetes, enfermedad pulmonar obstructiva, enfermedad renal crónica, enfermedad hepática crónica y con diversos grados de inmunodepresión producto de la malnutrición, enfermedades oncohematológicas, tratamientos con agentes inmunosupresores, trauma, cirugía y enfermedades infecciosas ${ }^{37-40}$. La mayoría de los estudios coinciden que la hipertensión arterial y la diabetes son los principales factores que se asocian a cuadros clínicos severos de COVID-19 y la obesidad es un factor de riesgo reconocido en Nueva York ${ }^{41,42}$.

Tabla 1. Grupos vulnerables.

\begin{tabular}{|c|c|c|}
\hline Características & $\begin{array}{c}\text { Richardson, et al. }{ }^{41} \\
(n=5700)\end{array}$ & $\begin{array}{c}\text { Zhang, et al. }{ }^{42} \\
(n=1464)\end{array}$ \\
\hline País (ciudad) & EEUU (Nueva York) & China (Wuhan) \\
\hline $\begin{array}{l}\text { Edad } \\
\text { Mediana (IQR*) }\end{array}$ & $63(52-75)$ & $64(49-70)$ \\
\hline \multicolumn{3}{|l|}{ Sexo } \\
\hline Femenino, n (\%) & $2263(39,7)$ & $728(49,7)$ \\
\hline Masculino, $\mathrm{n}(\%)$ & $3437(60,3)$ & $736(50,3)$ \\
\hline \multicolumn{3}{|l|}{ Comorbilidades, n (\%) } \\
\hline Hipertensión & $3026(56,6)$ & $306(20,9)$ \\
\hline Enfermedades Cardiovasculares & $966(18)$ & $211(14,4)$ \\
\hline Diabetes & $1808(33,8)$ & $211(14,4)$ \\
\hline Tumores malignos & $320(6)$ & $17(1,2)$ \\
\hline Enfermedades Crónicas respiratorias (EPOC**/Asma y otras) & $920(17,3)$ & $50(3,4)$ \\
\hline Enfermedad renal crónica & $454(8,5)$ & $27(1,8)$ \\
\hline Enfermedad hepática crónica & $30(0,6)$ & $36(2,5)$ \\
\hline Enfermedad cerebrovascular & $\mathrm{N} / \mathrm{R}$ & $47(3,2)$ \\
\hline Inmunodepresión (VIH/transplante) & $98(0,9)$ & $\mathrm{N} / \mathrm{R}^{* * *}$ \\
\hline Enfermedad metabólica (obesidad y otras) & $2528(60,7)$ & $\mathrm{N} / \mathrm{R}$ \\
\hline
\end{tabular}

*IQR (rango intercuartil). **EPOC (enfermedad pulmonar obstructiva). ***NR (no refiere).

Las manifestaciones más comunes son la fiebre, tos seca, mialgia, fatiga, anorexia y falta de aire; otros síntomas y signos de menor frecuencia son la cefalea, dolor de garganta, rinorrea, expectoración, hemoptisis, dolor torácico, dolor abdominal, náuseas, vómitos y diarrea $^{40,43-47}$. Las manifestaciones y complicaciones neurológicas derivadas de la afectación del Sistema Nervioso Central son el mareo, cefalea, deterioro de la conciencia, encefalopatía, encefalitis aguda, encefalopatía necrotizante hemorrágica aguda, accidente cerebrovascular, mielitis transversa, epilepsia y ataxia ${ }^{48,49}$. Por otra parte, las repercusiones clínicas originadas por invasión del Sistema Nervioso Periférico son la anosmia, hiposmia, ageusia, hipogeusia, neuralgia y síndrome de Guillain-Barré ${ }^{50,51}$. Sin embargo, las complicaciones neurológicas surgen como un área cada 298 vez más reconocida de morbilidad y mortalidad. En una cohorte de 179 pacientes con neumonía por SARSCoV-2, la enfermedad cerebrovascular preexistente también se asoció con una mayor mortalidad ${ }^{52}$. Una revisión sistemática y metanálisis también identificaron un riesgo 2,5 veces mayor de infección grave entre los pacientes con un accidente cerebrovascular previo ${ }^{53}$.

También se ha identificado como síntomas extrapulmonares de COVID-19 diversas lesiones cutáneas en el estudio efectuado por Galán, et al. en España, siendo los más frecuentes el exantema maculopapular o macular, erupción papulovesicular, pápulas dolorosas de color rojo púrpura acral con o sin vesículas (pseudo-sabañones) y la urticaria ${ }^{54}$. 
Las complicaciones asociadas al SARS-CoV-2 varían de acuerdo a los grupos etarios y comorbilidades asociadas a los pacientes, las cuales pueden repercutir en la inmunocompetencia de los individuos afectados. Las complicaciones más frecuentes observadas fueron el síndrome de distrés respiratorio agudo, arritmias, fallo cardíaco agudo y shock séptico; aunque está reportado otras alteraciones como el fallo renal agudo, fallo respiratorio, infección bacteriana secundaria, miocarditis aguda, infarto agudo de miocardio, trombosis venosa profunda, embolia pulmonar y coagulación intravascular diseminada (CID) $)^{42-47,55,56}$.

El estudio retrospectivo de Zhou, et al. identificaron varios factores de alta probabilidad de complicaciones, y muerte en particular en individuos de edad avanzada, niveles elevados de dímero-D, y una elevada puntuación de la evaluación del fallo orgánico secuencial (SOFA, por sus siglas en inglés) $)^{57}$. La susceptibilidad a padecer infecciones virales y complicaciones en adultos mayores, se debe en parte a la inmunosenescencia, que es consecuencia de los procesos intrínsecos que ocurren en el sistema inmune relacionados con el envejecimiento ${ }^{58,59}$. Estas características están relacionadas principalmente con la disminución en el número y actividad de las células de la inmunidad innata; involución tímica a partir de la pubertad que provoca un escaso repertorio de linfocitos vírgenes; con la senescencia replicativa que con conduce a un predominio de células de memoria y linfocitos exhaustos la cual es agravada por la estimulación antigénica crónica, principalmente a la infección por citomegalovirus (CMV) que finalmente lleva al desgaste progresivo del repertorio de linfocitos ${ }^{59,60}$.

Las manifestaciones clínicas producidas por SARSCoV-2 en niños son sustancialmente menos agresivas que en adultos, lo cual también ha sido descrito para SARS-CoV en el brote epidémico de $2002^{61}$. Los niños infectados pueden encontrarse asintomáticos o presentar fiebre, tos seca, fatiga, dolor de garganta congestión nasal, rinorrea, estornudos y síntomas gastrointestinales como dolor abdominal, náuseas, vómitos y diarrea con buen pronóstico, generalmente logrando la remisión del cuadro dentro de la primera o segunda semana del comienzo de la enfermedad ${ }^{62}$.

\section{Replicación y Patogénesis de SARS-CoV-2}

La entrada de SARS-CoV-2 a las células del hospedero está mediada por las subunidades S1 y S2 de la glicoproteína $\mathrm{S}^{63}$. La subunidad $\mathrm{S} 1$ contiene el motivo de unión al receptor (RBM, por sus siglas en inglés) que permite la unión del virión a su receptor, enzima convertidora de angiotensina 2 (ACE-2, por sus siglas en inglés) una carboxipeptidasa de membrana y S2 contiene los motivos estructurales que consisten en regiones de repeticiones de siete aminoácidos (HR1 y HR2, por sus siglas en inglés) implicadas en la fusión virus-membrana plasmática de las células epiteliales del hospedero del tracto respiratorio y digestivo, resultando en la endocitosis y liberación del genoma viral en el citoplasma celular ${ }^{63-65}$. Para la fusión de SARS-CoV-2 con la membrana de la célula es importante la escisión proteolítica en límite de la unión de la S1/S2 o S2 del homotrímero $\mathrm{S}$, por parte de la serinas proteasas transmembras 2 (TMPRSS2) asociadas a la superficie celular y catepsinas L y P que permiten la separación entre el RBM y el dominio de fusión ${ }^{63,67}$.

Una vez en el citoplasma de la célula del hospedero el genoma viral ARN monocatenario (+) no envuelto, comienza la traducción de dos largas réplicas de poliproteínas denominados ppla y pplab, las cuales son escindidas por las proteasas tipo papaína (PLpro), proteasa tipo serina y la proteasa principal generando las proteínas no estructurales (nsp1-nsp16) y el complejo replicasa-transcriptasa responsable de la replicación y transcripción del ARN subgenómicos ${ }^{67,68}$. Estos segmentos ribonucleicos actúan como ARNm, permitiendo la traducción de proteínas accesorias y proteínas estructurales $\mathrm{S}, \mathrm{E}, \mathrm{M}$ y $\mathrm{N}$ del virión con la participación de las subunidades ribosomales; estas últimas son introducidas en el retículo endoplasmático (RE) y el aparato de Golgi (AG) donde el genoma viral es encapsulado por las proteínas $\mathrm{N}$, finalmente se acoplan las demás proteínas estructurales y estos son liberados de los compartimentos RE-AG en forma de viriones maduros ${ }^{67,69}$. Por último, estas vesículas que contienen los viriones se fusionan con la membrana plasmática y se libera el SARS-CoV-2 maduro ${ }^{11,70}$.

La nueva evidencia planteada por Angeletti, et al. sugiere que la presión selectiva positiva, ha logrado modificaciones puntuales en aminoácidos de las proteínas no estructurales nsp2, y nsp3 que le confiere a SARS-CoV-2 mayor capacidad de propagación e infección en relación a SARS-CoV ${ }^{71}$. Esto puede ser relevante, ya que nsp3 está compuesto por múltiples dominios como PLpro que participa en la entrada del virus a la célula del hospedero, pero además nsp3 es un componente esencial del complejo replicasatranscriptasa, que es vital para su ciclo de vida ${ }^{72}$. Los diversos estudios realizados indican que SARS-CoV-2 tiene un efecto citopático directo en las células que 
infecta, a través mecanismos no establecidos, aunque su tasa de replicación elevada está implicada en la $\operatorname{misma}^{70,73}$.

\section{Respuesta inmune contra SARS-CoV-2}

\section{Respuesta inmune innata}

Las células epiteliales del tracto respiratorio son las primeras en ser infectadas y modular la respuesta inicial, ya que cuentan con receptores de reconocimiento de patrones (RRP) como los receptores tipo Toll (TLR-7 y 8), los receptores del gen I inducible por ácido retinoico (RIG-I, por sus siglas en inglés) y proteína 5 asociada a la diferenciación de melanoma (MDA5, por sus siglas en inglés) ambos de la familia de receptores tipo RIG-I, los cuales se encargan de reconocer el genoma de SARS-CoV-2 dentro la célula e iniciar la activación de los factores de transcripción IRF3, IRF7 y NF-kB ${ }^{74,75}$. Esto conduce a la liberación de interferones de tipo I (INF $\alpha$ e INF $\beta$ ) y mediadores inflamatorios como IL6 , TNF- $\alpha$, G-SCF y GM-SCF, pero además induce la producción de quimiocinas CXCL-8, CXCL-10 y CCL5 que facultan el reclutamiento de neutrófilos, células $\mathrm{NK}$, monocitos y linfocitos $\mathrm{T}$ al tracto respiratorio ${ }^{76}$. Las células epiteliales de la vía aérea producen factores antivirales como lactoferrina, proteínas surfactantes (SP-A y SP-D), $\beta$-defensinas humanas 1-4 y catelicidinas LL-37 que pueden interferir en ciclo vital de SARS-CoV-2 ${ }^{76-78}$. Por otra parte, el daño de las células endoteliales, la activación directa por $\mathrm{C} 3 \mathrm{a}$, C5a, IL-1, IL-6, TNF- $\alpha$ y SARS-CoV-2 de estas células en el parénquima pulmonar inducen la liberación de mediadores proinflamatorios, moléculas de adhesión (ICAM-1, VCAM-1, selectina P y E) y quimiocinas como CXCL-9, CXCL-10 y CCL-2 que favorecen la migración de células de la inmunidad innata $\mathrm{y}$ adquirida $^{79}$.

Los macrófagos alveolares, macrófagos diferenciados y neutrófilos reclutados, se encargan de la fagocitosis de células infectadas, cuerpos apoptóticos que contienen virus, partículas virales y viriones; liberan citocinas proinflamatorias (IL-1, IL-6, IL-8, IL12, IL-18, INF $\alpha$, INF $\beta$, TNF $\alpha$, G-CSF y GM-CSF), prostaglandinas, leucotrienos, enzimas proteolíticas e hidrolíticas, péptidos antimicrobianos (defensinas y catelicidinas), especies reactivas del oxígeno, óxido nítrico y diversidad de quimiocinas que se encargan del eliminación viral; aunque también contribuyen al daño alveolar difuso y edema pulmonar ${ }^{80}$. A pesar de la abundante infiltración de monocitos, neutrófilos y linfocitos, la respuesta inmune no es eficiente, ya que no existe linfocitos $\mathrm{T}$ altamente específicos contra SARS-
CoV-2 que puedan eliminar a las células infectadas y eviten la propagación del virus ${ }^{81,82}$. La acumulación de estas células en el pulmón provoca elevada producción citocinas proinflamatorias, que conducen a la tormenta de citocinas caracterizada por la liberación incontrolada de IL-1 $\beta$, IL-2, IL-6, IL-8, IL-10, TNF $\alpha$, G-CSF, GMCSF y MCP-1 (CCL-2) que provocan SDRA, fallo multiorgánico y elevación de parámetros inflamatorios ${ }^{83}$. La replicación activa y liberación de viriones maduros conduce a la activación del inflamasoma, que provoca la piroptosis en las infectadas con liberación de patrones moleculares asociados a daño (DAMPs, por sus siglas en inglés) a la circulación que son reconocidos por las células endoteliales, monocitos y macrófagos agravando la tormenta de citocinas ${ }^{84}$. (Figura 1) También la IL-6 puede unirse a la forma soluble de IL-6R (sIL-6R), formando un complejo con un dímero gp130, este complejo se deposita en la membrana de las células endoteliales favoreciendo la tormenta de citocinas que involucra mediadores inflamatorios mencionados ${ }^{85,86}$.

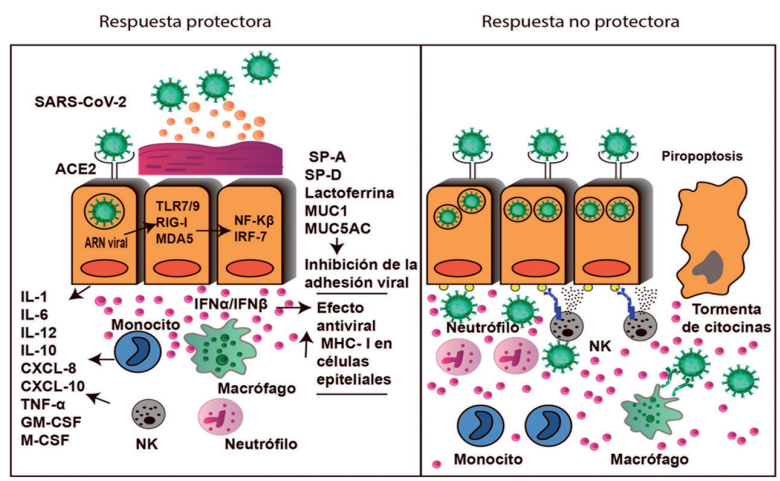

Figura 1. Respuesta inmune innata.

Respuesta protectora: inhibición de la adhesión viral por medio de SP-A, SP-D, lactoferrina, MUC1 y MUC5AC; secreción de citocinas y quimiocinas que atraen y activan células de la inmunidad innata, el macrófago secreta IFN $\alpha / \beta$, se incrementa la expresión de las MHC-I a nivel del macrófago y otras células. Respuesta no protectora: las células de inmunidad secretan grandes cantidades de citocinas y quimiocinas como la IL-1, IL-6, IL-12, IL-10, CXCL-8, CXCL-10, TNF- $\alpha$, GM-CFS, M-CSF (tormenta de citocinas); las células NK eliminan las células infectadas; los macrófagos inician la fagocitosis, todos estos mecanismos facilitan e incrementan el estado inflamatorio que provocan la piropoptosis.

Las células NK son las principales células de la inmunidad innata que se encargan de la eliminación viral, y ejercen su acción a través de la liberación de mediadores citotóxicos como granzima $\mathrm{B}$, perforinas, y serglicinas que activan la vía mitocondrial de la apoptosis en la célula infectada; expresan ligandos de receptores de muerte (Ligando del receptor de TNF, 
FASL y TRAIL) y liberan TNF $\alpha$ e INF $\gamma^{87-89}$. Esta última citocina induce una eficaz activación de los macrófagos, diferenciación de linfocitos $\mathrm{T}$ CD4+ vírgenes en células Th1 y promueve una respuesta por linfocitos T citotóxicos, por lo cual es esencial en la eliminación viral ${ }^{90}$. Sin embargo, se ha identificado un descenso significativo de células NK en pacientes con cuadros severos infectados con SARS-CoV-2, lo que sugiere el deterioro de la respuesta inmune antiviral ${ }^{91}$.

Las células dendríticas CD141+ presentes en la lámina propia del tracto respiratorio pueden ser activadas por el reconocimiento y endocitosis de las partículas virales de SARS-CoV-2 o por estimulación de citocinas proinflamatorias ${ }^{75,92}$. Estas células comienzan a madurar y migrar a los ganglios linfáticos regionales, donde activan eficazmente a los linfocitos T vírgenes CD4+ y CD $8+{ }^{75,93}$.

\section{Respuesta inmune adaptativa}

\section{Respuesta inmune humoral}

La respuesta humoral está mediada por los linfocitos $\mathrm{B}$, que reconocen directamente los antígenos virales de SARS-CoV-2, se activan y diferencian en células plasmáticas y células $\mathrm{B}$ de memoria ${ }^{94}$. Las primeras producen las inmunoglobulinas de la clase $\mathrm{M}, \mathrm{G}, \mathrm{y}$ A las cuales limitan la diseminación de los viriones en las células no infectadas, a través de mecanismos como la neutralización, opsonización, activación del complemento y la citotoxicidad celular dependiente de anticuerpos (ADCC, por sus siglas en inglés) ${ }^{94,95}$. No está claro la relevancia de la respuesta humoral en la contención y eliminación de SARS-CoV-2, pero los hallazgos de Zhao, et al. demuestran que la mediana del tiempo de seroconversión para IgM y IgG fue de 12 y 14 días respectivamente, con una frecuencia de seroconversión en 173 individuos se detectó en el $82,7 \% \quad \operatorname{IgM}(143 / 173)$ y solo el $64,7 \%$ de $\operatorname{IgG}$ $(112 / 173)^{96}$. Sin embargo, las investigaciones de Wang, et al. identificaron descensos significativos de los linfocitos B en sangre periférica de pacientes con neumonía moderada y severa, que pudiera sugerir un papel preponderante en la contención viral ${ }^{97}$. La importancia de la respuesta humoral también fue identificada por el estudio de Zhang, et al. donde encontraron IgG específica contra el RBM de la glicoproteína $\mathrm{S}$ de SARS-CoV-2, que limitó la propagación viral en niños infectados ${ }^{98}$. (Figura 2)

\section{Respuesta inmune celular}

Los linfocitos T cooperadores (Th) CD4+ en particular el subtipo Th-1, son importante para la eliminación de las células infectadas, estas células se encargan de la producción de IL-2, IL-12 e INF $\gamma$, siendo la IL-2 vital para la proliferación y diferenciación de los linfocitos B y T CD $8{ }^{+99}$. Los niveles elevados de IL-6 asociado TGF- $\beta$ polariza la diferenciación de los linfocitos Th vírgenes al subtipo Th-17 productor de IL-17A, IL-17F, IL-22 e IL-21 que agravan el proceso inflamatorio a nivel pulmonar y sistémico en pacientes con COVID-19 ${ }^{100}$.

La respuesta de linfocitos T citotóxicos (CTLs) es esencial para la completa resolución de la infección por SARS-CoV-2, ya que por distintos mecanismos que inducen la apoptosis de las células infectadas logra la remisión completa de los procesos infecciosos virales ${ }^{101}$. Los linfocitos T citotóxicos logran la eliminación de las células infectadas a través de mecanismos dependientes del contacto celular, donde intervienen la perforina, granzimas A, B, serglicina y FASL, los cuales activan las vías intrínseca y extrínseca de la apoptosis ${ }^{102,103}$. Pero además los CTLs secretan citocinas como el TNF $\alpha$ e INF $\gamma$, este último estimula a las células dendríticas a expresar IL-12 e induce la diferenciación de linfocitos $\mathrm{T}$ CD8+ vírgenes en células citotóxicas ${ }^{102,104}$.

Por los múltiples estudios realizados se ha logrado identificar una disminución significativa de las poblaciones de linfocitos T CD4+ y CD8+ en sangre periférica, principalmente en pacientes con cuadros clínicos moderados y severos de COVID-19 $9^{91,97,105}$.

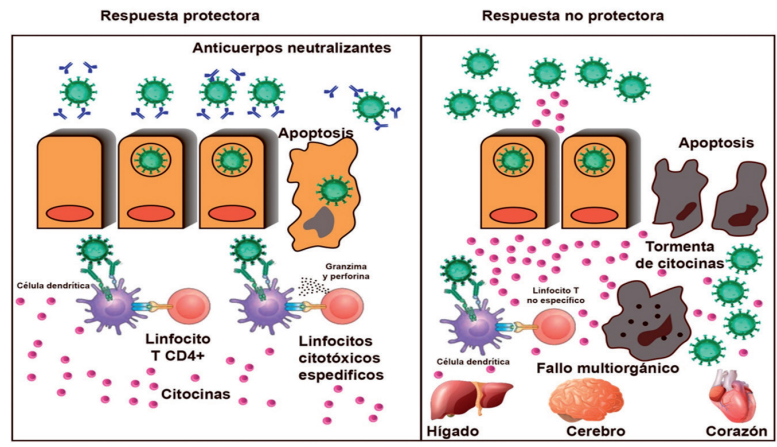

Figura 2. Respuesta inmune adaptativa.

Respuesta protectora: Neutralización viral mediada por IgG e IgA. La presentación de antígeno por las células dendríticas a los linfocitos T CD4+ y CD8+, esta última célula es específica para SARS-CoV-2, así eliminar a las células infectadas. Respuesta no protectora: La replicación viral continúa, incrementa la secreción de citocinas por los linfocitos $\mathrm{T}$ no específicos y ocurre la apoptosis en un mayor número de células, que todo esto conllevan a un fallo multiorgánico.

\section{Tratamiento de la COVID-19}

No existe un tratamiento antiviral específico recomendado para la COVID-19, y actualmente no existe vacuna disponible. El tratamiento es sintomático 
y la oxigenoterapia representa la principal intervención de tratamiento para pacientes con infección grave ${ }^{106}$. La ventilación mecánica puede ser necesaria en casos de insuficiencia respiratoria refractaria a la oxigenoterapia $^{107}$. El tratamiento de rescate con plasma convaleciente e inmunoglobulina $G$ por vía intravenosa (IgGIV) se administra a algunos casos críticos según sus condiciones ${ }^{108-110}$.

Se pueden desarrollar varias opciones para controlar o prevenir infecciones emergentes de SARS-CoV-2, incluidas vacunas, anticuerpos monoclonales, terapias basadas en oligonucleótidos, péptidos, terapias de interferón ${ }^{111}$. Sin embargo, es probable que las nuevas intervenciones requieran meses o años para desarrollarse. Dada la urgencia del brote de SARSCoV-2, nos centramos aquí en el potencial de reutilizar los agentes antivirales existentes aprobados o en desarrollo para tratar infecciones causadas por el virus de la inmunodeficiencia humana (VIH), el virus de la hepatitis B (VHB), el virus de la hepatitis C (VHC) y la influenza, según la experiencia terapéutica con otras dos infecciones causadas por coronavirus humanos: SARS-CoV y MERS-CoV ${ }^{112,113}$.

Tabla 2. Fármacos aprobados y ensayos clínicos contra el SARS-CoV-2.

\begin{tabular}{|c|c|c|c|}
\hline Drogas & Mecanismo de acción & $\begin{array}{c}\text { Enfermedades en que se aprueba } \\
\text { su uso }\end{array}$ & Ref. \\
\hline Lopinavir/Ritonavir & $\begin{array}{l}\text { Inhibe las proteasas de VIH-1 y VIH-2. También } \\
\text { inhibe a la Proteasa de cisteína tipo 3-quimiotripsina } \\
\text { (3CLpro) }\end{array}$ & $\begin{array}{l}\text { VIH/SIDA, SARS-CoV y MERS- } \\
\mathrm{CoV}\end{array}$ & 114,115 \\
\hline $\begin{array}{l}\text { Cloroquina e } \\
\text { Hidroxicloroquina }\end{array}$ & Incremento del $\mathrm{pH}$ endosomal y de fagolisosoma & $\begin{array}{l}\text { Malaria y enfermedades } \\
\text { autoinmunes }\end{array}$ & 116,117 \\
\hline Remdesivir & $\begin{array}{l}\text { Análogo de nucleósido que inhibe a la ARN } \\
\text { polimerasa dependiente de ARN viral, interfiriendo } \\
\text { con la replicación viral }\end{array}$ & Ébola ensayo clínico fase II/III & $118,119,120$ \\
\hline Tocilizumab & $\begin{array}{l}\text { Bloquea al receptor de IL- } 6 \text {, inhibiendo la } \\
\text { transducción de señales }\end{array}$ & $\begin{array}{l}\text { Tormenta de citocinas asociado } \\
\text { al tratamiento con CAR-T, artritis } \\
\text { reumatoide }\end{array}$ & 121,122 \\
\hline Ruxolitinib & $\begin{array}{l}\text { Inhibidor selectivo de las quinasas asociadas a } \\
\text { Janus (JAK1 y JAK2). Estas quinasas median la } \\
\text { transducción de señales de citocinas }\end{array}$ & Tormenta de citocinas & 123 \\
\hline Siltuximab & $\begin{array}{l}\text { Se une de forma específica a la IL- } 6 \text { y así evita la } \\
\text { unión de esta citocina a sus receptores }\end{array}$ & $\begin{array}{l}\text { Enfermedad de Castleman } \\
\text { multicéntrica }\end{array}$ & 124,125 \\
\hline Baricitinib & $\begin{array}{l}\text { Inhibe las Janus quinasas (JAK1 y JAK2) de forma } \\
\text { transitoria y reversible }\end{array}$ & Artritis reumatoide & 126 \\
\hline Interferón $\alpha-2 B$ & $\begin{array}{l}\text { Induce en las células un estado de resistencia a } \\
\text { infecciones virales, y modula la fase efectora del } \\
\text { sistema inmune para neutralizar los virus o eliminar } \\
\text { células infectadas }\end{array}$ & $\begin{array}{ll}\text { Papilomatosis } & \text { respiratoria } \\
\text { recurrente, condiloma } & \text { acuminado, } \\
\text { hepatitis B y C. } & \end{array}$ & 127 \\
\hline Ivermectina & $\begin{array}{l}\text { Inhibe la importación de la integrasa, la ivermectina } \\
\text { se adhiere al heterodímero } \alpha / \beta 1 \text { importina (IMP) }\end{array}$ & $\begin{array}{l}\text { Antiparasitario: ectoparasitosis, } \\
\text { contra nemátodos y oncocercosis }\end{array}$ & 128,129 \\
\hline Colchicina & $\begin{array}{l}\text { Inhibe el inflamasoma en dos niveles: inhibe la } \\
\text { activación del receptor } \mathrm{P} 2 \mathrm{x} 7 \text { y la polimerización de } \\
\text { ASC, inhibiendo así la interacción entre dominios } \\
\text { similares a la pirina. }\end{array}$ & Gota y fiebre mediterránea familiar & 130 \\
\hline
\end{tabular}

\section{Conclusión}

La infección por SARS-CoV-2 es un problema de salud global con repercusiones socioeconómicas a nivel mundial. Los orígenes de este agente no están totalmente establecidos, ya que no se ha identificado al huésped intermediario en su modo de transmisión al hombre. Las manifestaciones clínicas dependen del estatus inmunológico del individuo infectado, las comorbilidades asociadas, el grupo etario y las particularidades patogénicas de SARS-CoV-2. Por otro lado, la inmunosenescencia del adulto mayor, las enfermedades crónicas y cualquier factor que conduzca a una inmunodepresión se asocian a complicaciones en pacientes con COVID-19. En las primeras etapas de la infección de SARS-CoV-2 la respuesta inmune innata 
se encarga de la contención y eliminación viral mediada por neutrófilos, monocitos, macrófagos, mastocitos, células NK y el patrón inflamatorio generado a nivel pulmonar y sistémico. Este mismo estado inflamatorio genera daño en el parénquima pulmonar, y en diversos órganos asociado al efecto citopático directo de SARSCoV-2. Para la completa resolución de este proceso se requiere, una respuesta inmune adaptativa altamente específica y robusta contra este agente.

Los linfocitos Th-1 y CTLs son esenciales para la completa eliminación de SARS-CoV-2 en las células infectadas. Pero se ha descrito en pacientes graves bajos niveles de linfocitos B, linfocitos T CD4+ y CD8+, aunque se ha identificado un aumento del subtipo Th-17 que agrava el proceso inflamatorio sistémico. No existen estudios acerca del papel de los linfocitos NKT, linfocitos $\mathrm{T} \gamma \delta$, células linfoides innatas, células dendríticas plasmocitoides, linfocitos $\mathrm{T}$ reguladores, y otros por lo que la dinámica de la respuesta inmune frente a SARS-CoV-2 no está esclarecida. Actualmente no existe tratamiento específico para COVID-19, ya que existe resultados contradictorios en los ensayos clínicos que evaluaron algunos fármacos.

\section{Conflicto de Interés}

Los autores declaran que no tienen conflicto de intereses.

\section{Referencias}

1. Guo Y-R, Cao Q-D, Hong Z-S, Tan Y-Y, Chen $\mathrm{S}-\mathrm{D}$, Jin H-J, et al. The origin, transmission and clinical therapies on coronavirus disease 2019 (COVID-19) outbreak - an update on the status. Mil Med Res. 2020; 7(1): 11. doi: 10.1186/s40779020-00240-0

2. Gorbalenya AE, Baker SC, Baric RS, de Groot RJ, Drosten C, Gulyaeva AA, et al. The species Severe acute respiratory syndrome-related coronavirus: Classifying 2019-nCoV and naming it SARSCoV-2. Nat Microbiol. 2020; 5(4): 536-544. doi: 10.1038/s41564-020-0695-Z

3. Lai C-C, Shih T-P, Ko W-C, Tang H-J, Hsueh P-R. Severe acute respiratory syndrome coronavirus 2 (SARS-CoV-2) and coronavirus disease-2019 (COVID-19): The epidemic and the challenges. Int J Antimicrob Agents. 2020; 55(3): 105924. doi:10.1016/j.ijantimicag.2020.105924

4. Cucinotta D, Vanelli M. WHO Declares COVID-19 a Pandemic. Acta Biomed. 2020; 91(1): 157-160 pp. doi: org/10.23750/abm.v91i1.9397

5. Paules CI, Marston HD, Fauci AS. Coronavirus
Infections-More than just the common cold. JAMA. 2020; 323(8): 707-708. doi:10.1001/ jama.2020.0757

6. Schoeman D, Fielding BC. Coronavirus envelope protein: current knowledge. Virol J. 2019; 16(1): 69. doi: 10.1186/s12985-019-1182-0

7. King AMQ, Adams MJ, Carstens EB, Lefkowitz EJ, editors. Virus Taxonomy. Ninth report of the International Committee on Taxonomy of Virues. San Diego: Elsevier; 2012. p. 796-805.

8. Cui J, Li F, Shi Z-L. Origin and evolution of pathogenic coronaviruses. Nat Rev Microbiol. 2019; 17(3): 181-192. doi: 10.1038/s41579-0180118-9

9. Corman VM, Muth D, Niemeyer D, Drosten C. Hosts and sources of endemic human coronaviruses. Adv Virus Res. 2018; 100: 163188. doi: 10.1016/bs.aivir.2018.01.001

10. Gralinski LE, Menachery VD. Return of the Coronavirus: 2019-nCoV. Viruses. 2020; 12(2): 135. doi: $10.3390 / \mathrm{v} 12020135$

11. Xinyi Y, Ng Y, Tam J, Liu D. Human Coronaviruses: a review of virus-host interactions. Diseases. 2016; 4: 26. doi: 10.3390/diseases4030026

12. 12. Raoult D, Zumla A, Locatelli F, Ippolito Kroemer G. Coronavirus infections: Epidemiological, clinical and immunological features and hypotheses. Cell Stress. 2020; 2020; 4(4): 66-75. doi: 10.15698/cst2020.04.216

13. Song Z, Xu Y, Bao L, Zhang L, Yu P, Qu Y, et al. From SARS to MERS, thrusting coronaviruses into the spotlight. Viruses. 2019; 11(1): 59. doi: 10.3390/v11010059

14. Shereen MA, Khan S, Kazmi A, Bashir N, Siddique R. COVID-19 infection: Origin, transmission, and characteristics of human coronaviruses. J Adv Res. 2020; 24: 91-98. doi: 10.1016/j.jare.2020.03.005

15. Ul Qamar MT, Alqahtani SM, Alamri MA, Chen L-L. Structural basis of SARS-CoV-2 3CLpro and anti-COVID-19 drug discovery from medicinal plants. J Pharm Anal. 2020; Epub ahead of print. doi: 10.1016/j.jpha.2020.03.009

16. Andersen KG, Rambaut A, Lipkin WI, Holmes EC, Garry RF. The proximal origin of SARS-CoV-2. Nat Med. 2020; 26(4): 450-452. doi: 10.1038/ s41591-020-0820-9

17. Ge H, Wang X, Yuan X, Xiao G, Wang C, Deng T, et al. The epidemiology and clinical information about COVID-19. Eur J Clin Microbiol Infect Dis. 2020; 1-9. doi: 10.1007/s10096-020-03874-z

18. Lu H, Stratton CW, Tang Y-W. Outbreak of pneumonia of unknown etiology in Wuhan, China: The mystery and the miracle. J Med Virol. 2020; 
92(4): 401-402. doi:10.1002/jmv.25678

19. Song H-D, Tu C-C, Zhang G-W, Wang S-Y, Zheng K, Lei L-C, et al. Cross-host evolution of severe acute respiratory syndrome coronavirus in palm civet and human. Proc Natl Acad Sci U.S.A. 2005; 102(7): 2430-2435. doi: 10.1073/ pnas.0409608102

20. Ye Z-W, Yuan S, Yuen K-S, Fung S-Y, Chan C-P, Jin D-Y. Zoonotic origins of human coronaviruses. Int J Biol Sci. 2020; 16(10): 1686-1697. doi: 10.7150/ijbs. 45472

21. Zhang Y-Z, Holmes EC. A Genomic perspective on the origin and emergence of SARS-CoV-2. Cell. 2020; 181(2): 223-227. doi: 10.1016/j. cell.2020.03.035

22. Sohrabi C, Alsafi Z, O'Neill N, Khan M, Kerwan A, Al-Jabir A, et al. World Health Organization declares global emergency: A review of the 2019 novel coronavirus (COVID-19). Int J Surg. 2020; 76: 71-76. doi: org/10.1016/j.ijsu.2020.02.034

23. Memish ZA, Almasri M, Turkestani A, Al-Shangiti AM, Yezli S. Etiology of severe communityacquired pneumonia during the 2013 Hajj-part of the MERS-CoV surveillance program. Int $\mathrm{J}$ Infect Dis. 2014; 25: 186-190. doi: 10.1016/j. ijid.2014.06.003

24. Karadag E. Increase in COVID-19 cases and case-fatality and case-recovery rates in Europe: a cross-temporal meta-analysis. J Med Virol. 2020: 10.1002/jmv.26035. doi: 10.1002/jmv.26035

25. Li Q, Guan X, Wu P, Wang X, Zhou L, Tong Y, et al. Early transmission dynamics in Wuhan, China, of novel Coronavirus-Infected Pneumonia. N Engl J Med. 2020; 382(13): 1199-1207. doi: 10.1056/ NEJMoa2001316

26. Chan JF-W, Yuan S, Kok K-H, To KK-W, Chu $\mathrm{H}$, Yang J, et al. A familial cluster of pneumonia associated with the 2019 novel coronavirus indicating person-to-person transmission: A study of a family cluster. Lancet. 2020; 395(10223): 514-523. doi: 10.1016/S0140-6736(20)30154-9

27. Yu P, Zhu J, Zhang Z, Han Y. A Familial cluster of infection associated with the 2019 novel coronavirus indicating possible person-to-person transmission during the incubation period. J Infect Dis. 2020; 221(11): 1757-1761. doi: 10.1093/ infdis/jiaa077

28. van Doremalen N, Bushmaker T, Morris DH, Holbrook MG, Gamble A, Williamson BN, et al. Aerosol and surface stability of SARSCoV-2 as compared with SARS-CoV-1. N Engl J Med. 2020; 382(16): 1564-1567. doi: 10.1056/ NEJMc2004973
29. Dong L, Tian J, He S, Zhu C, Wang J, Liu C, et al. Possible vertical transmission of sars-cov-2 from an infected mother to her newborn. JAMA. 2020; 323(18): 1846-1848 doi:10.1001/jama.2020.4621

30. Chen Y, Peng H, Wang L, Zhao Y, Zeng L, Gao $\mathrm{H}$, et al. Infants born to mothers with a new coronavirus (COVID-19). Front Pediatr. 2020; 8(104). doi: 10.3389/fped.2020.00104

31. Karimi-Zarchi M, Neamatzadeh H, Dastgheib SA, Abbasi H, Mirjalili SR, Behforouz A, et al. Vertical transmission of coronavirus disease 19 (COVID-19) from infected pregnant mothers to neonates: A review. Fetal Pediatr Pathol. 2020; 1-5. doi: 10.1080/15513815.2020.1747120

32. Chen H, Guo J, Wang C, Luo F, Yu X, Zhang W, et al. Clinical characteristics and intrauterine vertical transmission potential of COVID-19 infection in nine pregnant women: A retrospective review of medical records. Lancet. 2020; 395(10226): 809815. doi: 10.1016/S0140-6736(20)30360-3

33. Long Q-X, Tang X-J, Shi Q-L, Li Q, Deng H-J, Yuan $\mathrm{J}$, et al. Clinical and immunological assessment of asymptomatic SARS-CoV-2 infections. Nat Med. 2020. doi:10.1038/s41591-020-0965-6

34. Ren L, Gonzalez R, Xu J, Xiao Y, Li Y, Zhou H, et al. Prevalence of human coronaviruses in adults with acute respiratory tract infections in Beijing, China. J Med Virol. 2011; 83(2): 291-297. doi: 10.1002/jmv.21956

35. Petrosillo N, Viceconte G, Ergonul O, Ippolito G, Petersen E. COVID-19, SARS and MERS: are they closely related? Clin Microbiol Infect. 2020; 26(6): 729-734. doi: 10.1016/j.cmi.2020.03.026

36. Wang $\mathrm{Y}$, Wang $\mathrm{Y}$, Chen $\mathrm{Y}$, Qin Q. Unique epidemiological and clinical features of the emerging 2019 novel coronavirus pneumonia (COVID-19) implicate special control measures. J Med Virol. 2020; 92(6): 568-576. doi: doi. org/10.1002/jmv.25748

37. Guo W, Li M, Dong Y, Zhou H, Zhang Z, Tian C, et al. Diabetes is a risk factor for the progression and prognosis of COVID-19. Diabetes Metab Res Rev. 2020: e3319. doi: 10.1002/dmrr.3319

38. Wang X, Fang X, Cai Z, Wu X, Gao X, Min J, et al. Comorbid chronic diseases and acute organ injuries are strongly correlated with disease severity and mortality among COVID-19 patients: A systemic review and meta-analysis. Research (Wash DC). 2020; 2020: 2402961. doi: $10.34133 / 2020 / 2402961$

39. Chinen J, Shearer WT. Secondary immunodeficiencies, including HIV infection. J Allergy Clin Immunol. 2010; 125(2 Suppl 2): 
S195-S203. doi: 10.1016/j.jaci.2009.08.040

40. Raifman MA, Raifman JR, Julia R Raifman. Disparities in the population at risk of severe illnes from COVID-19 by income. Am J Prev Med. 2020; 59(1): 137-139. doi: 10.1016/j. amepre.2020.04.003

41. Richardson S, Hirsch JS, Narasimhan M, Crawford JM, McGinn T, Davidson KW, et al. Presenting characteristics comorbidities, and outcomes among 5700 patients hospitalized with COVID-19 in the New York. JAMA. 2020; 323(20): 20522059. doi: 10.1001/jama.2020.6775

42. Zhang J, Wang X, Jia X, Li X, Hu K, Chen G, et al. Risk factors for Disease severity, unimprovement, and mortality in COVID-19 patients in Wuhan, China. Clin Microbiol Infect. 2020; 26(6): 767772. doi: 10.1016/j.cmi.2020.04.012

43. Hassan SA, Sheikh FN, Jamal S, Ezeh JK, Akhtar A. Coronavirus (COVID-19): a review of clinical features, diagnosis, and treatment. Cureus. 2020; 12(3): e7355-e. doi: 10.7759/cureus.7355

44. Zhang J, Wang M, Zhao M, Guo S, Xu Y, Ye J, et al. The Clinical characteristics and prognosis factors of mild-moderate patients with COVID-19 in a mobile cabin hospital: A retrospective, singlecenter study. Front Public Health. 2020; 8(264). doi: 10.3389/fpubh.2020.00264

45. Wang D, Hu B, Hu C, Zhu F, Liu X, Zhang J, et al. Clinical characteristics of 138 hospitalized patients with 2019 novel Coronavirus-Infected pneumonia in Wuhan, China. JAMA. 2020; 323(11): 10611069. doi: $10.1001 /$ jama.2020.1585

46. Chen N, Zhou M, Dong X, Qu J, Gong F, Han Y, et al. Epidemiological and clinical characteristics of 99 cases of 2019 novel coronavirus pneumonia in Wuhan, China: A descriptive study. Lancet. 2020; 395(10223): 507-13. doi: 10.1016/S01406736(20)30211-7

47. Xu X-W, Wu X-X, Jiang X-G, Xu K-J, Ying L-J, Ma $\mathrm{C}-\mathrm{L}$, et al. Clinical findings in a group of patients infected with the 2019 novel coronavirus (SARSCov-2) outside of Wuhan, China: Retrospective case series. BMJ. 2020; 368: m606. doi: 10.1136/ bmj.m792

48. Ahmad I, Azam Rathore F. Neurological manifestations and complications of COVID-19: A literature review. J Clin Neurosci. 2020; 77: 8-12. doi: 10.1016/j.jocn.2020.05.017

49. Das G, Mukherjee N, Ghosh S. Neurological Insights of COVID-19 Pandemic. ACS Chem Neurosci. 2020; 11(9): 1206-1209. doi: 10.1021/ acschemneuro.0c00201
50. Butowt R, Bilinska K. SARS-CoV-2: Olfaction, brain infection, and the urgent need for clinical samples allowing earlier virus detection. ACS Chem Neurosci. 2020; 11(9): 1200-1203. doi:10.1021/acschemneuro.0c00172

51. Zhao H, Shen D, Zhou H, Liu J, Chen S. GuillainBarré syndrome associated with SARS-CoV-2 infection: causality or coincidence? Lancet Neurol. 2020; 19(5): 383-384. doi: 10.1016/ S1474-4422(20)30109-5

52. Du R-H, Liang L-R, Yang C-Q, Wang W, Cao T-Z, $\mathrm{Li}$ M, et al. Predictors of mortality for patients with COVID-19 pneumonia caused by SARS-CoV-2: A prospective cohort study. Eur Respir J. 2020; 55(5): 2000524. doi: 10.1183/13993003.005242020

53. Aggarwal G, Lippi G, Henry BM. Cerebrovascular disease is associated with an increased disease severity in patients with coronavirus disease 2019 (COVID-19): A pooled analysis of published literature. Int J Stroke. 2020; 15(4): 385-389. doi: 10.1177/1747493020921664

54. Galván Casas C, Català A, Carretero Hernández G, Rodríguez-Jiménez P, Fernández Nieto D, Rodríguez-Villa Lario A, et al. Classification of the cutaneous manifestations of COVID-19: A rapid prospective nationwide consensus study in Spain with 375 cases. Br J Dermatol. 2020; 183(1): 7177. doi: doi.org/10.1111/bjd.19163

55. Terpos E, Ntanasis-Stathopoulos I, Elalamy I, Kastritis E, Sergentanis TN, Politou M, et al. Hematological findings and complications of COVID-19. Am J Hematol. 2020; 95(7): 834-847. doi: 10.1002/ajh.25829

56. Long B, Brady WJ, Koyfman A, Gottlieb M. Cardiovascular complications in COVID-19. Am J Emerg Med. 2020; 38(7): 1504-1507. doi: 10.1016/j.ajem.2020.04.048

57. Zhou F, Yu T, Du R, Fan G, Liu Y, Liu Z, et al. Clinical course and risk factors for mortality of adult inpatients with COVID-19 in Wuhan, China: A retrospective cohort study. Lancet. 2020; 395(10229): 1054-1062. doi: 10.1016/S01406736(20)30566-3

58. Pawelec G. Age and immunity: what is "immunosenescence"? Exper Gerontol. 2017; 105: 4-9. doi: 10.1016/j.exger.2017.10.024

59. Oh S-J, Lee JK, Shin OS. Aging and the immune system: The Impact of immunosenescence on viral infection, immunity and vaccine immunogenicity. Immune Netw. 2019; 19(6): e37-e. doi: 10.4110/ in.2019.19.e37 
60. Pawelec G. Hallmarks of human "immunosenescence": adaptation or dysregulation? Immun Ageing. 2012; 9(1): 15. doi: 10.1186/17424933-9-15

61. Principi N, Bosis S, Esposito S. Effects of coronavirus infections in children. Emerg Infect Dis. 2010; 16(2): 183-188. doi: 10.321/ eid1602.090469

62. Mustafa NM, A Selim L. Characterisation of COVID-19 pandemic in paediatric age group: A systematic review. J Clin Virol. 2020; 104395. doi: 10.1016/j.jcv.2020.104395

63. Walls AC, Park Y-J, Tortorici MA, Wall A, McGuire AT, Veesler D. Structure, function, and antigenicity of the SARS-CoV-2 spike glycoprotein. Cell. 2020; 181(2): 281-292. doi: 10.1016/j.cell.2020.02.058

64. Letko M, Marzi A, Munster V. Functional assessment of cell entry and receptor usage for SARS-CoV-2 and other lineage B betacoronaviruses. Nat Microbiol. 2020; 5(4): 562-569. doi: 10.1038/s41564-020-0688-y

65. Wrapp D, Wang N, Corbett KS, Goldsmith JA, Hsieh C-L, Abiona O, et al. Cryo-EM structure of the 2019-nCoV spike in the prefusion conformation. Science. 2020; 367(6483): 12601263. doi: 10.1126/science.abb2507

66. Wan Y, Shang J, Graham R, Baric RS, Li F. Receptor recognition by the novel coronavirus from Wuhan: an analysis based on decade-long structural studies of SARS Coronavirus. J Virol. 2020; 94(7): e00127-20. doi: 10.1128/JVI.0012720

67. de Wilde AH, Snijder EJ, Kikkert M, van Hemert MJ. Host factors in coronavirus replication. Curr Top Microbiol Immunol. 2018; 419: 1-42. doi: 10.1007/82_2017_25

68. Yang D, Leibowitz JL. The structure and functions of coronavirus genomic 3' and 5' ends. Virus Res. 2015; 206: 120-133. doi: 10.1016/j. virusres.2015.02.025

69. Fehr AR, Perlman S. Coronaviruses: an overview of their replication and pathogenesis. Methods Mol Biol. 2015; 1282: 1-23. doi: 10.1007/978-14939-2438-7_1

70. Kumar S, Nyodu R, Maurya VK, Saxena SK. Morphology, genome organization, replication, and pathogenesis of severe acute respiratory syndrome Coronavirus 2 (SARS-CoV-2). In: Saxena SK, editor. Coronavirus Disease 2019 (COVID-19). Singapore: Springer Singapore; 2020; 23-31. doi: 10.1007/978-981-15-4814-7_3
71. Angeletti S, Benvenuto D, Bianchi M, Giovanetti M, Pascarella S, Ciccozzi M. COVID-2019: The role of the nsp2 and nsp3 in its pathogenesis. J Med Virol. 2020; 92(6): 584-588

72. Lei J, Kusov Y, Hilgenfeld R. Nsp3 of coronaviruses: Structures and functions of a large multi-domain protein. Antiviral Res. 2018; 149: 58-74. doi: 10.1002/jmv.25719

73. Cagno V. SARS-CoV-2 cellular tropism. Lancet Microbe. 2020; 1(1): e2-e3. doi: 10.1016/S26665247(20)30008-2

74. Leiva-Juárez MM, Kolls JK, Evans SE. Lung epithelial cells: therapeutically inducible effectors of antimicrobial defense. Mucosal Immunol. 2018; 11(1): 21-34. doi: 10.1038/mi.2017.71

75. Li G, Fan Y, Lai Y, Han T, Li Z, Zhou P, et al. Coronavirus infections and immune responses. $\mathrm{J}$ Med Virol. 2020; 92(4): 424-432. doi: 10.1002/ jmv. 25685

76. Newton AH, Cardani A, Braciale TJ. The host immune response in respiratory virus infection: balancing virus clearance and immunopathology. Semin Immunopathol. 2016; 38(4): 471-482. doi: 10.1007/s00281-016-0558-0

77. Hiemstra PS, Mc Cray PB, Jr. Bals R. The innate immune function of airway epithelial cells in inflammatorylung disease. EurRespirJ.2015;45(4): 1150-1162. doi: 10.1183/09031936.00141514

78. Weitnauer M, Mijošek V, Dalpke AH. Control of local immunity by airway epithelial cells. Mucosal Immunol. 2016; 9(2): 287-298. doi: 10.1038/ mi.2015.126

79. Konradt C, Hunter CA. Pathogen interactions with endothelial cells and the induction of innate and adaptive immunity. Eur J Immunol. 2018; 48(10): 1607-1620. doi: 10.1002/eji.201646789

80. Gordon SB, Read RC. Macrophage defences against respiratory tract infections: The immunology of childhood respiratory infections. Br Med Bull British. 2002; 61(1): 45-61. doi: 10.1093/bmb/61.1.45

81. Xu Z, Shi L, Wang Y, Zhang J, Huang L, Zhang C, et al. Pathological findings of COVID-19 associated with acute respiratory distress syndrome. Lancet Respir Med. 2020; 8(4): 420-422. doi: 10.1016/ S2213-2600(20)30076-X

82. Liang Y, Wang M-L, Chien C-S, Yarmishyn AA, Yang Y-P, Lai W-Y, et al. Highlight of immune pathogenic response and hematopathologic effect in SARS-CoV, MERS-CoV, and SARS-Cov-2 Infection. Front Immunol. 2020; 11(1022). doi: 10.3389/fimmu.2020.01022 
83. 83. Tisoncik JR, Korth MJ, Simmons CP, Farrar J, Martin TR, Katze MG. Into the eye of the cytokine storm. Microbiol Mol Biol Rev. 2012; 76(1): 1632. doi: 10.1128/MMBR.05015-11

84. Tay MZ, Poh CM, Rénia L, MacAry PA, Ng LFP. The trinity of COVID-19: immunity, inflammation and intervention. Nat Rev Immunol. 2020; 20(6): 363-374. doi: 10.1038/s41577-020-0311-8

85. Zhao M. Cytokine storm and immunomodulatory therapy in COVID-19: Role of chloroquine and anti-IL-6 monoclonal antibodies. Int J Antimicrob Agents. 2020; 55(6) 105982. doi: 10.1016/j. ijantimicag.2020.105982

86. Moore JB, June CH. Cytokine release syndrome in severe COVID-19. Science. 2020; 368(6490): 473-474. doi: 10.1126/science.abb8925

87. Abel AM, Yang C, Thakar MS, Malarkannan S. natural killer cells: development, maturation, and clinical utilization. Front Immunol. 2018; 9: 1869. doi: 10.3389/fimmu.2018.01869

88. Waggoner SN, Reighard SD, Gyurova IE, Cranert SA, Mahl SE, Karmele EP, et al. Roles of natural killer cells in antiviral immunity. Curr Opin Virol. 2016; 16: 15-23. doi: 10.1016/j.coviro.2015.10.008

89. Prager I, Watzl C. Mechanisms of natural killer cell-mediated cellular cytotoxicity. J Leukoc Biol. 2019; 105(6): 1319-1329. doi: 10.1002/jlb. mr0718-269r.

90. Kang S, Brown HM, Hwang S. Direct antiviral mechanisms of interferon-gamma. Immune Netw. 2018; 18(5): e33-e. doi: 10.4110/in.2018.18.e33.

91. He R, Lu Z, Zhang L, Fan T, Xiong R, Shen X, et al. The clinical course and its correlated immune status in COVID-19 pneumonia. J Clin Virol. 2020;127:104361. doi: 10.1016/j.jcv.2020.104361

92. Silvin A, Yu CI, Lahaye X, Imperatore F, Brault $\mathrm{J}-\mathrm{B}$, Cardinaud S, et al. Constitutive resistance to viral infection in human CD141+ dendritic cells. Sci Immunol. 2017; 2(13): eaai8071. doi: 10.1126/ sciimmunol.aai8071

93. Bedoui $\mathrm{S}$, Gebhardt $\mathrm{T}$. Interaction between dendritic cells and $\mathrm{T}$ cells during peripheral virus infections: a role for antigen presentation beyond lymphoid organs? Curr Opin Immunol. 2011; 23(1): 124-130. doi: 10.1016/j.coi.2010.11.001

94. Kumar S, Nyodu R, Maurya VK, Saxena SK. Host immune response and immunobiology of human SARS-CoV-2 Infection. Coronavirus Disease 2019 (COVID-19). 2020: 43-53. doi: 10.1007/978-98115-4814-7_5

95. di Mauro G, Cristina S, Concetta R, Francesco R, Annalisa C. SARS-Cov-2 infection: Response of human immune system and possible implications for the rapid test and treatment. Int Immunopharmacol. 2020; 84: 106519. doi: 10.1016/j.intimp.2020.106519

96. Zhao J, Yuan Q, Wang H, Liu W, Liao X, Su Y, et al. Antibody responses to SARS-CoV-2 in patients of novel coronavirus disease 2019. Clin Infect Dis. 2020: ciaa344. doi: 10.1093/cid/ciaa344.

97. Wang F, Nie J, Wang H, Zhao Q, Xiong Y, Deng L, et al. Characteristics of Peripheral Lymphocyte Subset Alteration in COVID-19 Pneumonia. J Infect Dis. 2020; 221(11): 1762-1769. doi: 10.1093/infdis/jiaa150

98. Zhang Y, Xu J, Jia R, Yi C, Gu W, Liu P, et al. Protective humoral immunity in SARS-CoV-2 infected pediatric patients. Cell Mol Immunol. 2020. doi: 10.1038/s41423-020-0438-3

99. Astuti I, Ysrafil. Severe Acute respiratory syndrome Coronavirus 2 (SARS-CoV-2): An overview of viral structure and host response. Diabetes Metab Syndr. 2020; 14(4): 407-412. doi: 10.1016/j.dsx.2020.04.020

100. Wu D, Yang XO. TH17 responses in cytokine storm of COVID-19: An emerging target of JAK2 inhibitor Fedratinib. J Microbiol Immunol Infect. 2020; 3(3): 368-370. doi: 10.1016/j. jmii.2020.03.005

101. Janice Oh H-L, Ken-En Gan S, Bertoletti A, Tan Y-J. Understanding the $\mathrm{T}$ cell immune response in SARS coronavirus infection. Emerg Microbes Infect. 2012; 1(9): e23-e. doi: 10.1038/emi.2012.26

102. Channappanavar R, Zhao J, Perlman S. T cellmediated immune response to respiratory coronaviruses. Immunol Res. 2014; 59(1-3): 118128. doi: 10.1007/s12026-014-8534-z

103. Chávez-Galán L, Arenas-Del Angel MC, Zenteno E, Chávez R, Lascurain R. Cell death mechanisms induced by cytotoxic lymphocytes. Cell Mol Immunol. 2009; 6(1): 15-25. doi: 10.1038/ cmi.2009.3

104. Ito H, Seishima M. Regulation of the induction and function of cytotoxic $\mathrm{T}$ lymphocytes by natural killer T cell. J Biomed Biotechnol. 2010; 2010: 641757. doi: 10.1155/2010/641757

105. Jiang M, Guo Y, Luo Q, Huang Z, Zhao R, Liu S, et al. $T$ cell subset counts in peripheral blood can be used as discriminatory biomarkers for diagnosis and severity prediction of COVID-19. J Infect Dis. 2020; 222(2): 198-202: doi: 10.1093/infdis/ jiaa252

106. Peng F, Tu L, Yang Y, Hu P, Wang R, Hu Q, et al. Management and treatment of COVID-19: The Chinese experience. Can J Cardiol. 2020; 36(6): 915-930. doi: 10.1016/j.cjca.2020.04.010 
107. 107. Cunningham AC, Goh HP, Koh D. Treatment of COVID-19: old tricks for new challenges. Crit Care. 2020; 24(1): 91. doi: 10.1186/s13054-0202818-6

108. Zhang B, Liu S, Tan T, Huang W, Dong Y, Chen $\mathrm{L}$, et al. Treatment with convalescent plasma for critically Ill patients with SARS-CoV-2 infection. Chest. 2020; 158(1): e9-e13. doi: 10.1016/j. chest.2020.03.039

109. Ye M, Fu D, Ren Y, Wang F, Wang D, Zhang F, et al. Treatment with convalescent plasma for COVID-19 patients in Wuhan, China. J Med Virol. 2020. doi: 10.1002/jmv.25882

110. Nguyen AA, Habiballah SB, Platt CD, Geha RS, Chou JS, McDonald DR. Immunoglobulins in the treatment of COVID-19 infection: Proceed with caution! Clin Immunol. 2020; 216 108459. doi: 10.1016/j.clim.2020.108459

111. Li L, Li R, Wu Z, Yang X, Zhao M, Liu J, et al. Therapeutic strategies for critically ill patients with COVID-19. Ann Intensive Care. 2020; 10(1): 45. doi: 10.1186/s13613-020-00661-z

112. Li H, Liu S-M, Yu X-H, Tang S-L, Tang C-K. Coronavirus disease 2019 (COVID-19): current status and future perspectives. Int $\mathrm{J}$ Antimicrob Agents. 2020: 105951. doi: 10.1016/j. ijantimicag.2020.105951

113. Jean S-S, Lee P-I, Hsueh P-R. Treatment options for COVID-19: The reality and challenges. J Microbiol Immunol Infect. 2020; 53(3): 436-443. doi: $10.1016 /$ j.jmii.2020.03.034

114. Chu CM, Cheng VCC, Hung IFN, Wong MML, Chan KH, Chan KS, et al. Role of lopinavir/ ritonavir in the treatment of SARS: initial virological and clinical findings. Thorax. 2004; 59(3): 252. doi: 10.1136/thorax.2003.012658

115. Choy K-T, Wong AY-L, Kaewpreedee P, Sia SF, Chen D, Hui KPY, et al. Remdesivir, lopinavir, emetine, and homoharringtonine inhibit SARSCoV-2 replication in vitro. Antiviral Res. 2020; 178: 104786. doi: 10.1016/j.antiviral.2020.104786

116. Colson P, Rolain J-M, Lagier J-C, Brouqui P, Raoult D. Chloroquine and hydroxychloroquine as available weapons to fight COVID-19. Int J Antimicrob Agents. 2020; 55(4): 105932. doi: 10.1016/j.ijantimicag.2020.105932

117. Gautret P, Lagier J-C, Parola P, Hoang VT, Meddeb L, Sevestre J, et al. Clinical and microbiological effect of a combination of hydroxychloroquine and azithromycin in 80 COVID-19 patients with at least a six-day follow up: A pilot observational study. Travel Med Infect Dis. 2020; 34: 101663. doi: 10.1016/j.tmaid.2020.101663
118. Grein J, Ohmagari N, Shin D, Diaz G, Asperges E, Castagna A, et al. Compassionate Use of Remdesivir for Patients with Severe Covid-19. N Engl J Med. 2020; 382(24): 2327-2336. doi: 10.1056/NEJMoa2007016.

119. Wang Y, Zhang D, Du G, Du R, Zhao J, Jin Y, et al. Remdesivir in adults with severe COVID-19: a randomised, double-blind, placebo-controlled, multicentre trial. Lancet. 2020; 395(10236): 15691578. doi: 10.1016/S0140-6736(20)31022-9

120. Tchesnokov EP, Feng JY, Porter DP, Götte M. Mechanism of inhibition of ebola virus rnadependent RNA polymerase by Remdesivir. Viruses. 2019; 11(4): 326. doi: 10.3390/v11040326

121. Zhang C, Wu Z, Li J-W, Zhao H, Wang G-Q. The cytokine release syndrome (CRS) of severe COVID-19 and Interleukin-6 receptor (IL-6R) antagonist Tocilizumab may be the key to reduce the mortality. Int J Antimicrob Agents. 2020; 55(5): 105954. doi: 10.1016/j.ijantimicag.2020.105954

122. Le RQ, Li L, Yuan W, Shord SS, Nie L, Habtemariam BA, et al. FDA Approval summary: Tocilizumab for treatment of chimeric antigen receptor $\mathrm{T}$ Cellinduced severe or life-threatening cytokine release syndrome. Oncologist. 2018; 23(8): 943-947. doi: 10.1634/theoncologist.2018-0028

123. Stebbing J, Phelan A, Griffin I, Tucker C, Oechsle O, Smith D, et al. COVID-19: combining antiviral and anti-inflammatory treatments. Lancet Infect Dis. 2020; 20(4): 400-402. doi: 10.1016/S14733099(20)30132-8

124. Jamilloux Y, Henry T, Belot A, Viel S, Fauter M, El Jammal T, et al. Should we stimulate or suppress immune responses in COVID-19? Cytokine and anti-cytokine interventions. Autoimmun Rev. 2020; 19(7): 102567. doi: 10.1016/j.autrev.2020.102567

125. Gritti G, Raimondi F, Ripamonti D, Riva I, Landi F, Alborghetti L, et al. Use of siltuximab in patients with COVID-19 pneumonia requiring ventilatory support. medRxiv. 2020; 20048561. doi: 10.1101/2020.04.01.20048561

126. Cantini F, Niccoli L, Matarrese D, Nicastri E, Stobbione P, Goletti D. Baricitinib therapy in COVID-19: A pilot study on safety and clinical impact. J Infect. 2020; Epub ahead of print. doi: 10.1016/j.jinf.2020.04.017

127. Sallard E, Lescure F-X, Yazdanpanah Y, Mentre F, Peiffer-Smadja N. Type 1 interferons as a potential treatment against COVID-19. Antiviral research. 2020; 178: 104791. doi: 10.1016/j. antiviral.2020.104791

128. Caly L, Druce JD, Catton MG, Jans DA, Wagstaff KM. The FDA-approved drug ivermectin 
inhibits the replication of SARS-CoV-2 in vitro. Antiviral Res. 2020; 178: 104787. doi: 10.1016/j. antiviral.2020.104787

129. Bray M, Rayner C, Noël F, Jans D, Wagstaff K. Ivermectin and COVID-19: A report in antiviral research, widespread interest, an FDA warning, two letters to the editor and the authors' responses. Antiviral Res. 2020: 104805. doi: 10.1016/j. antiviral.2020.104805

130. Deftereos SG, Siasos G, Giannopoulos G, Vrachatis DA, Angelidis C, Giotaki SG, et al. The Greek study in the effects of colchicine in COvid-19 complications prevention (GRECCO-19 study): Rationale and study design. Hellenic J Cardiol. 2020; S1109-9666(20)30061-0. doi: 10.1016/j. hjc. 2020.03.002 